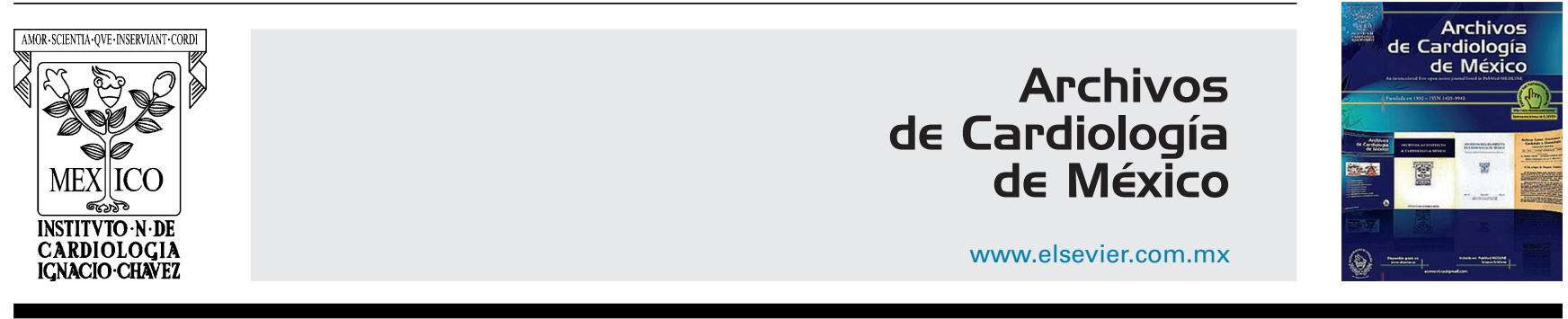

FE DE ERRORES

\title{
Fe de errores de «Tratamiento exitoso de disección aórtica en paciente embarazada: procedimiento de Bentall y hemiarco»
}

\section{Erratum to «Successful treatment of aortic dissection in pregnant patient: Bentall procedure and hemiarch»}

\author{
Luis Raul Meza-López ${ }^{a}$, Luis Efren Santos-Martínez ${ }^{\text {,** }}$, Silvia Hernández-Meneses ${ }^{a}$, \\ Juan Ignacio Moreno-Valencia ${ }^{a}$, Jesus Zaín Campos-Larios ${ }^{c}$, \\ Nielzer Armando Rodríguez-Almendros ${ }^{b}$, Victor Manuel Lozano-Torres ${ }^{a}$ \\ y Moisés Cuttiel Calderón-Abbo ${ }^{d}$
}

\footnotetext{
a Departamento de Cirugía Cardiotorácica, Unidad Médica de Alta Especialidad, Hospital de Cardiología del Centro Médico Nacional, Siglo XXI, Instituto Mexicano del Seguro Social, D.F., México

${ }^{\mathrm{b}}$ Departamento de Hipertensión Pulmonar y Función Ventricular Derecha, Unidad Médica de Alta Especialidad, Hospital de Cardiología del Centro Médico Nacional, Siglo XXI, Instituto Mexicano del Seguro Social, D.F., México

c Departamento de Cuidados Intensivos Posquirúrgicos Cardiovasculares, Unidad Médica de Alta Especialidad, Hospital de Cardiología del Centro Médico Nacional, Siglo XXI, Instituto Mexicano del Seguro Social, D.F., México

¿ Dirección General, Unidad Médica de Alta Especialidad, Hospital de Cardiología del Centro Médico Nacional, Siglo XXI, Instituto Mexicano del Seguro Social, D.F., México
}

En el artículo titulado Tratamiento exitoso de disección aórtica en paciente embarazada: procedimiento de Bentall y hemiarco se ha detectado un error en la autoría. Se sustituye al autor Gilberto Pérez-Rodríguez por el autor Moisés Cuttiel Calderón-Abbo, quedando la autoría de la siguiente manera:

Luis Raul Meza-López ${ }^{\mathrm{a}}$, Luis Efren Santos-Martínez ${ }^{\mathrm{b}, *}$, Silvia Hernández-Meneses ${ }^{\mathrm{a}}$, Juan Ignacio Moreno-Valencia ${ }^{\mathrm{a}}$, Jesus Zaín Campos-Larios ${ }^{c}$, Nielzer Armando Rodríguez-Almendros ${ }^{b}$, Victor Manuel Lozano-Torres ${ }^{a}$ y Moisés Cuttiel Calderón-Abbo ${ }^{d}$

Véase contenido relacionado en DOI: http://dx.doi.org/10.1016/j.acmx.2015.04.004

* Autor para correspondencia.

Correo electrónico: luis.santosma@imss.gob.mx (L.E. Santos-Martínez). 